\title{
TERAPIA DE ACEPTACIÓN Y COMPROMISO EN UN CASO DE TRAS- TORNO MIXTO DE ANSIEDAD Y DEPRESIÓN EN UNA PACIENTE CON CÁNCER
}

\author{
ACCEPTANCE AND COMMITMENT THERAPY IN A CASE OF ADJUSTMENT \\ DISORDER WITH MIXED ANXIETY AND DEPRESSED MOOD IN A PATIENT WITH \\ CANCER
}

\author{
Paula Fernanda Pérez Rivero y Ana Fernanda Uribe Rodríguez \\ Universidad Pontificia Bolivariana Bucaramanga. Bucaramanga, Colombia.
}

Resumen

Se expone el caso de una paciente con diagnóstico de cáncer de mama quien había desarrollado un trastorno mixto de ansiedad y depresión con conductas de evitación. La paciente presentaba síntomas clínicos importantes relacionados con estados depresivos (anhedonia, insomnio, Ilanto, aislamiento, desórdenes alimenticios, pensamientos de desesperanza y minusvalía), así como síntomas de ansiedad tales como activación fisiológica, sensación de intranquilidad y pensamientos catastróficos. Sumado a esto presentaba un patrón de evitación conductual hacia todos los estímulos que ella consideraba podían alterarla. Desde el enfoque de la terapia de aceptación y compromiso se propuso como objetivo terapéutico la aceptación de la enfermedad y del malestar emocional que ella estaba experimentando; también se planteó la recuperación de las esferas vitales, siguiendo como punto de partida sus propios valores. El tratamiento se llevó a cabo en 14 sesiones y se diseñó implementando las técnicas de la Terapia de Aceptación y Compromiso dentro de las cuales se incluyeron la desesperanza creativa, la desactivación de las funciones verbales, la clarificación de valores y la pérdida de control sobre los eventos privados. En los resultados se observó un cambio positivo en las conductas de la paciente, así como
Abstract

This paper introduces the case of a woman with breast cancer who had developed a mixed depressive-anxiety disorder with avoidance behaviors. The patient presented depressive symptoms like listlessness, insomnia, weeping, food disorders and hopelessness though. Also, she exhibited physiological arousal and restlessness feelings. Additionally, the patient had an avoidance patron behavior in relation with all stimulus she believed could hurt her. Based on the Acceptance and Commitment Therapy (ACT) the intervention planted the following objectives: the patient will learn to accept her illness and the emotional distress that she was experiencing, also, the patient will recover the other areas of her life that she had abandoned. The treatment was developed in 14 sessions. The therapist used these techniques: creative hopelessness, disabling verbal functions, values clarification and loss of control over private events. In the results, it was observed a positive change in the behavior of the patient as well as a decrease in emotional distress that was his reason for initial consultation.

Keywords: Acceptance and Commitment Therapy, depression, evaluation, treatment, cancer.

Correspondencia:

Paula Fernanda Pérez Rivero; Ana Fernanda Uribe Rodríguez

Facultad de Psicología Universidad Pontificia Bolivariana Seccional Bucaramanga.

Campus Universitario Km. 7 Vía a Piedecuesta Bucaramanga, Colombia.

E-mail: paula.perez@upb.edu.co; anafernanda.uribe@upb.edu.co 


\section{una disminución del malestar emocional que constituía su motivo de consulta inicial.}

Palabras clave: Terapia de aceptación y compromiso, depresión, evaluación, intervención, cáncer.

\section{INTRODUCCIÓN}

El tratamiento de los problemas psicológicos en personas con diagnóstico de cáncer ha sido abordado a partir de diferentes enfoques terapéuticos, algunos de los cuales plantean como objetivo la eliminación o reducción de síntomas que causan malestar emocional(1). Según la literatura, con alguna frecuencia se observa la presencia de trastorno adaptativo en pacientes oncológicos ${ }^{(2)}$. Otros autores, han señalado que en ocasiones el cáncer puede cursar con trastornos en el estado de ánimo ${ }^{(3)}$, de ansiedad $^{(4)}$ y en algunos casos, con trastornos que incluyen alteraciones del pensamiento y la percepción ${ }^{(5)}$. Se ha observado que la comorbilidad del cáncer con trastornos mentales empeora la calidad de vida de los pacientes y que está asociada a un peor pronóstico de la enfermedad ${ }^{(6)}$. En este sentido, el tratamiento de las condiciones psicológicas asociadas al cáncer se ha propuesto como uno de los objetivos de la intervención integral y multidisciplinar de pacientes oncológicos $^{(7)}$. Lo anterior, se justifca además, en evidencias empíricas, que sugieren que sí se observa mejoría en el bienestar emocional y el estado de salud general en pacientes oncológicos que han recibido intervención y/o acompañamiento terapéutico $^{(8)}$. El modelo cognitivo-conductual, señala que el fortalecimiento de estrategias de afrontamiento, la reestructuración de creencias irracionales y la reducción de síntomas clínicos, les permite a los pacientes adaptarse con mayor facilidad a los cambios derivados de la enfermedad y su tratamiento ${ }^{(9)}$.
Por otra parte, desde la perspectiva de la terapia de aceptación y compromiso (ACT), se afirma que los problemas psicológicos de los pacientes responden a la evitación experiencial, un patrón de conducta en el cual se esquivan todos los estímulos relacionados con el cáncer, porque les causan malestar emocional y físico ${ }^{(10)}$. Dicha estrategia resulta muy útil al principio, porque disminuye en gran medida las sensaciones y estados indeseables, sin embargo, provoca a largo plazo la restricción de experiencias significativas y reforzantes para la vida de los pacientes, y la consecuente reaparición de las alteraciones emocionales ${ }^{(11)}$. Como respuesta a lo anterior, ACT es un modelo terapéutico que interviene sobre las conductas de evitación experiencial, dirigiendo a los pacientes a mantener sus esferas vitales activas ${ }^{(12)}$.

En este sentido ACT se revela como una alternativa de tratamiento eficaz en pacientes afectados o bloqueados tras el diagnóstico de cáncer y sus consecuencias $^{(13)}$. ACT mejora la calidad de vida de los pacientes promoviendo actuaciones acordes a los valores del paciente. Promueve la flexibilidad psicológica al alterar el patrón de evitación destructiva que le sitúa en una lucha infructuosa contra los propios sentimientos y sentimientos, alejándole de sus metas valiosas ${ }^{(14)}$. Los métodos clínicos de ACT se dirigen a clarificar los valores personales, contactar con la ineficacia de la lucha contra el malestar, y a aceptar los temores, preocupaciones o recuerdos del paciente de cáncer, orientándole al aquí y el ahora y a la acción como vía para volver a vivir una vida valiosa y 
con sentido aun con las limitaciones que se vayan encontrando en el proceso de enfermedad e incluso con una corta expectativa de vida ${ }^{(15)}$.

Este enfoque terapéutico ha tenido resultados eficaces en la intervención de pacientes con cáncer tal y como lo reportan diversos estudios con distintos tipos de diagnósticos de cáncer ${ }^{(10,13-15)}$. Los resultados de dichas investigaciones han mostrado una mayor repercusión de la intervención basada en ACT, especialmente cuando se tienen en cuenta los cambios a largo plazo (al año de seguimiento). También es importante señalar que los cambios de la condición de ACT se centran en la activación conductual aún en presencia de malestar.

Con base en lo anterior, el propósito de este estudio de caso es presentar la intervención psicológica desde el modelo de ACT en una paciente con cáncer de mama.

\section{Caso clínico}

Mujer de 35 años con diagnóstico de cáncer de mama estadio II (en adelante LP) quien presentaba problemas emocionales caracterizados por sintomatología ansiosa y depresiva y en la cual se observaron conductas de evitación experiencial. Anteriormente había sido diagnosticada con depresión durante la adolescencia y luego a los 27 años, para lo cual recibió tratamiento psiquiátrico. Actualmente, convive con su hijo de 5 años de edad, trabaja como taxista y en ocasiones se dedica al comercio. Su red de apoyo social se ha visto disminuida debido sus conductas de evitación. Sus objetivos para la intervención consistían en "alejar" los pensamientos relacionados con la enfermedad y la "depresión", no "llorar tanto" y "desaparecer la ansiedad". Cuando se le preguntó por las estrategias intentadas hasta el momento para eliminar los pensamientos y los sentimientos, refi- rió que evitaba realizar actividades que la "pusieran mal" como las diligencias médicas, hablar con otras personas: "Siempre me preguntan cómo estoy y esto me pone mal". También afirmó que cuando empezaba a "sentirse deprimida" se quedaba en cama todo el día viendo televisión en compañía de su hijo.

A partir de la evaluación se encontró que la paciente tenía las siguientes conductas problema: 1) Aislamiento social: Evitaba relacionarse con las demás personas. Esta sola, había perdido su red de relaciones sociales y no había intentado iniciar nuevas relaciones. Presentaba temor ante la posibilidad de fracasar en las relaciones interpersonales. 2) Apatía: Desinterés por las cosas, no encontraba disfrute en las actividades que realizaba. No hablaba con las demás personas de otros temas diferentes a sus quejas. 3) Autoimagen y auto-concepto deteriorados: Tenía constantes quejas sobre su aspecto físico, sobre su incapacidad para resolver, se refería de forma inferior respecto de sí misma en relación con otros, 4) Patrón de evitación conductual: Evitaba las diligencias y procedimientos médicos. Evitaba hablar de cosas que la hicieran sufrir o le provocaran malestar psicológico. Evitaba las responsabilidades cotidianas. Permanecía en cama todo el día, 5) Quejas verbales: Constantemente aludía a los "problemas terribles que tenía", se evidenciaba un discurso monotemático en torno a su sufrimiento personal, 6) Pensamientos de minusvalía e indefensión: Se presentaban de manera constante y eran evocados principalmente ante la presencia de una situación que requería afrontamiento: yo no soy capaz de nada" "por qué será que nadie me quiere" "yo no soy capaz de hablar con la gente, no sé cómo hacer, creo que no voy a encajar" y 7) Ansiedad: Respuesta fisiológica (taquicardia, sudoración, tensión muscular), intranquilidad, desasosiego. 


\section{Diseño}

Diseño tipo $\mathrm{ABA}^{(16)}$, en el cual se estableció una línea de base a través de los instrumentos seleccionados y de los criterios conductuales establecidos. A continuación se introdujo el tratamiento indicado para cada conducta problema y luego, se realizó una retirada de la práctica del tratamiento durante la sesión. Para la medición de las conductas objetivo se llevaron a cabo auto registros y aplicación de las escalas clínicas. Para analizar los datos, se llevó a cabo un tratamiento descriptivo a través de gráficas, en las cuales se pudo observar el cambio en las conductas diana ${ }^{(17)}$.

\section{Evaluación}

Se llevó a cabo una entrevista en la cual se recolectó la información relacionada con las conductas problema de la paciente y se realizó una evaluación de la conducta dentro de la consulta. Para complementar el reporte verbal y la observación se aplicaron los siguientes instrumentos: Inventario de depresión de Beck-II, adaptación española (BDI-II) ${ }^{(18)}$. Escala de Ansiedad y Depresión Hospitalaria (HAD), validada para población colombiana ${ }^{(19)}$, Escala de ansiedad Estado-Rasgo (STAI E-A) ${ }^{(20)}$. Otros instrumentos que fueron utilizados como recurso terapéutico y a su vez como indicadores de seguimiento fueron: el Cuestionario de Aceptación y Acción, adaptación española ${ }^{(21)}$, el Registro de Malestar y Acciones Valiosas, traducido al español (22), el Formulario Narrativo de Valores traducido al español (22), y el Cuestionario de Valores traducido al español ${ }^{(22)}$.

\section{Tratamiento}

Basándose en la Terapia de Aceptación y Compromiso la intervención psicológica se encaminó a promover el cambio contextual y la aceptación psicológica. En coherencia con esto se propusieron los siguientes indicadores de seguimiento: a) Verbalizaciones que hicieran referencia a la ansiedad, pensamientos, preocupaciones, recuerdos cuya topografía indicara alguna forma de aceptación o distanciamiento. b) Verbalizaciones que hicieran referencia a la ansiedad, recuerdos cuya topografía no indicara alguna forma de aceptación o distanciamiento, c) La implicación en cuestiones prácticas que situaran a LP en la dirección de alcanzar sus objetivos en la vida seleccionadas por ella misma en función de sus valores, d) La mejora de la calidad de vida de la paciente en general. El detalle de las intervenciones se muestra en la tabla 1.

\section{Resultados}

Al finalizar la intervención, la paciente logró aceptar su enfermedad y los pensamientos y emociones asociados a ésta e implicarse en las cuestiones que ella valoraba como importantes en su vida: Relaciones familiares, actividad laboral y educación de su hijo. La evolución es puesta de manifiesto en verbalizaciones como: "Aunque a veces me siento mal, sé que estoy bien y puedo hacer las cosas que me gustan y que valen la pena", o "sé que el cáncer está ahí, es algo que me sucedió, pero eso no significa que estoy acabada".

Para realizar un seguimiento de la evolución de la paciente se tomaron dos clases de registros: 1) Evaluaciones periódicas con instrumentos estandarizados, en el cual se monitoreaban los síntomas depresivos y de ansiedad por los cuales acudió la paciente a consulta, pues si bien el objetivo de la terapia no estaba encaminado a la disminución de estos eventos privados si se pretendía la mejora de la calidad de vida de LP, y; 2) Registro de indicadores conductuales. A continuación se presentan los resultados de estas categorías. 
Tabla 1. Intervención por sesiones basada en la Terapia de Aceptación y Compromiso

\begin{tabular}{|c|c|c|c|}
\hline Bloque & Técnicas & Sesiones & Indicadores \\
\hline \multirow{3}{*}{$\begin{array}{l}\text { Desesperanza } \\
\text { creativa }\end{array}$} & Metáfora el campo de hoyos & 2,4 & \multirow{3}{*}{$\begin{array}{l}\text { Conducta verbal de } \\
\text { reconocimiento de áreas } \\
\text { vitales descuidadas }\end{array}$} \\
\hline & Ejercicio el funeral & 2 & \\
\hline & Metáfora quitarse las gafas & 8 & \\
\hline \multirow{3}{*}{$\begin{array}{l}\text { Clarificación de } \\
\text { valores }\end{array}$} & Metáfora del Jardín & 3,10 & \multirow{3}{*}{$\begin{array}{l}\text { Listado de valores con } \\
\text { acciones dirigidas a } \\
\text { objetivos vitales }\end{array}$} \\
\hline & Establecer compromisos & $\begin{array}{l}3,4,5,10 \\
11,12,13 \\
14\end{array}$ & \\
\hline & Cuestionario de Valores & 3 & \\
\hline \multirow{4}{*}{$\begin{array}{l}\text { El control es el } \\
\text { problema }\end{array}$} & Si deseas quitar algo lo tendrás & 4,10 & \multirow{4}{*}{$\begin{array}{l}\text { Disminución de } \\
\text { conductas de control } \\
\text { y evitación de eventos } \\
\text { privados }\end{array}$} \\
\hline & Metáfora El Polígrafo & 3 & \\
\hline & Metáfora Alimentar al tigre & 3,6 & \\
\hline & Ejercicio del reflejo rotuliano & 6 & \\
\hline \multirow{8}{*}{$\begin{array}{l}\text { Desliteralización } \\
\text { de funciones } \\
\text { verbales }\end{array}$} & Metáfora las dos escalas & 5 & \multirow{8}{*}{$\begin{array}{l}\text { Aumento de acciones } \\
\text { orientadas a valores } \\
\text { Disminución del control } \\
\text { verbal sobre la conducta }\end{array}$} \\
\hline & Metáfora La Caja & 6 & \\
\hline & Ejercicio ¿Cuáles son los números? & 5 & \\
\hline & Metáfora mensaje en el computador & 6 & \\
\hline & Metáfora el tablero de ajedrez & 7 & \\
\hline & Metáfora casa con nubes & 8 & \\
\hline & Metáfora saltar en paracaídas & 8 & \\
\hline & Ejercicio de repetir palabras & 9 & \\
\hline \multirow{5}{*}{$\begin{array}{l}\text { Compromiso } \\
\text { y cambio de } \\
\text { conducta }\end{array}$} & Metáfora del viaje & 12,13 & \multirow{5}{*}{$\begin{array}{l}\text { Verbalizaciones de } \\
\text { aceptación y cambio } \\
\text { Mantenimiento y } \\
\text { aumento de las acciones } \\
\text { orientadas a valores. }\end{array}$} \\
\hline & $\begin{array}{l}\text { Ejercicio de cambiar la forma de } \\
\text { coger el lápiz }\end{array}$ & 10 & \\
\hline & Metáfora viaje y punto en el horizonte & 11 & \\
\hline & Metáfora del Jinete & 11 & \\
\hline & Evaluación de acciones a valores & $\begin{array}{c}9,10,12 \\
13,14\end{array}$ & \\
\hline
\end{tabular}

En el BDI-Il se observó una reducción de la a severidad de la depresión pasando de depresión moderada a no significativa clínicamente. En las dos primeras mediciones (sesiones 1 y 4) LP obtuvo puntajes de 24 y 20, lo cual corresponde a depresión moderada, en la tercera evaluación, se obtuvo un puntaje de 12, lo cual indicaba depresión leve y en el último registro se obtuvo un puntaje de 8 , el cual no indica significancia clínica.
Con relación al STAI se administró a la paciente en la primera sesión y en otras tres adicionales de seguimiento. Los resultados mostraron que la sub-escala de Ansiedad Rasgo se mantuvo relativamente estable a lo largo de las mediciones, mientras que en la de Ansiedad Estado los puntajes fueron disminuyendo pasando de una categoría clínica a una no clínica (Ver la figura 1). 
Figura 1. Seguimiento de los síntomas de ansiedad en la Escala de Ansiedad Estado/Rasgo STAI E-R

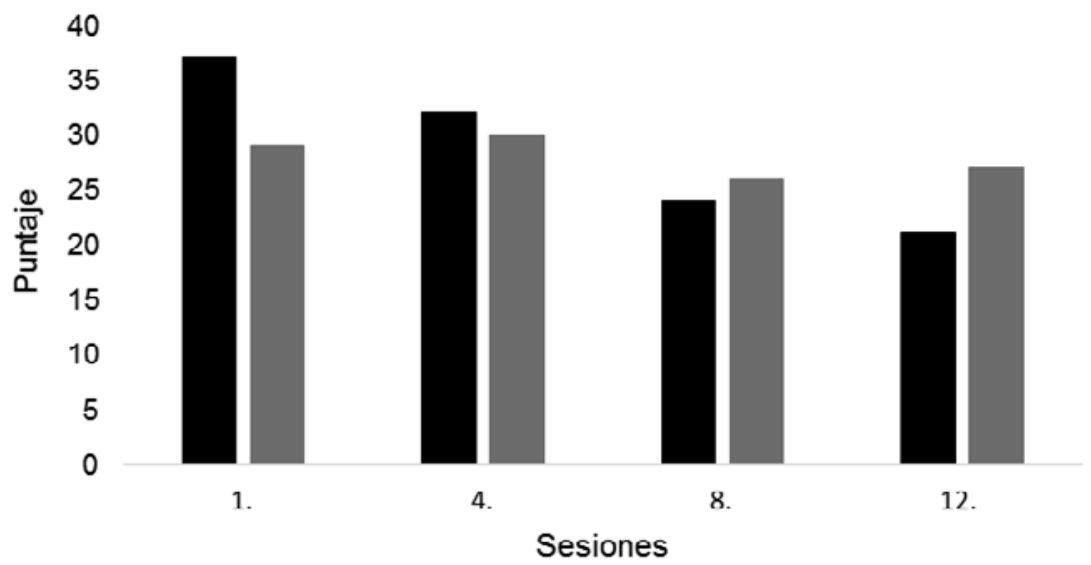

Ansiedad estado

Ansiedad rasgo

De igual forma, se administró el HAD. Los resultados observados en la HAD coinciden con los hallados en el BDI-II y en el STAI, en los cuales también se muestra una disminución de los síntomas de ansiedad y depresión. En la escala de ansiedad se redujo la puntuación desde 10 hasta 5 y en la escala de depresión desde 11 hasta 6 .

Para establecer el grado de diferencia encontrado entre las escalas clínicas, se realizó un Análisis de Varianza de una Vía (ANOVA) entre la primera y la últi- ma evaluación encontrándose diferencias estadísticamente significativas en todas las escalas. Ver la tabla 2.

Resultados de seguimiento por indicadores conductuales

Uno de los principales problemas de la paciente era la intrusión de pensamientos negativos (indefensión y minusvalía) que según ella la "llevaban a sentirse mal" y a no "hacer nada" aquí se presentaba una fusión entre los eventos

Tabla 2. Resultados pre y post de las escalas clínicas

\begin{tabular}{|l|c|c|c|}
\hline Escala & Pre $(\mathrm{M})$ & Post $(\mathrm{M})$ & $p$ \\
\hline BDI-II & 1 & 0 & $<0,001^{* *}$ \\
\hline STAI-Rasgo & 2 & 1 & $0,04^{*}$ \\
\hline STAI- Estado & 2 & 0 & $0,02^{*}$ \\
\hline HAD-Ansiedad & 3 & 1 & $0,001^{* *}$ \\
\hline HAD-Depresión & 3 & 0 & $<0,001^{* *}$ \\
\hline
\end{tabular}

BD-II; Inventario de Depresión de Beck-II; STAI: Escala de ansiedad Estado-Rasgo; HAD: Escala de Ansiedad y Depresión Hospitalaria. Nota. $M=$ Media. ${ }^{*} p<0,05 ;{ }^{* *} p<0,001$ 
privados y las actividades externas de la paciente, por lo cual se implementó una estrategia de distanciamiento según el modelo de ACT. Para realizar un seguimiento de esta conducta problema se le indicó a la paciente que llevara un autoregistro de la cantidad de pensamientos intrusivos que se le presentaban en una semana y de las veces que estos pensamientos "no le permitían realizar sus actividades" y también que registrara las veces que había experimentado los pensamientos sin que éstos interfirieran con su agenda del día. Los cuatro primeros registros (sesiones: 2 a 5) corresponden únicamente a la evaluación de los pensamientos (Línea de base). Los registros consecutivos corresponden al período de intervención (sesiones 6 a 11) y los últimos 3 corresponden a la retirada del tratamiento. Antes de iniciar el tratamiento LP reportada una cantidad elevada de pensamientos que le impedían realizar sus actividades y a medida que fue avanzado el proceso de la terapia estos pensamientos disminuyeron mientras que se nota un aumento de pensamientos no interferentes (Ver la figura 2).

Finalmente, para realizar un seguimiento sobre las acciones que estaba realizando la paciente respecto de sus esferas vitales se le indicó que llevará un registro de las tareas que iba ejecutando que se orientaban a conseguir sus valores y también que registrara las conductas de evitación. Los cuatro primeros registros (sesiones: 2 a 5) corresponden únicamente a la evaluación de los pensamientos (Línea de base). Los registros consecutivos corresponden al período de intervención (sesiones 6 a 11) y los últimos 3 corresponden a la retirada del tratamiento (ver la figura 3 ).

Como puede verse, a medida que iba transcurriendo el proceso terapéutico la paciente fue ampliando su repertorio

\section{Figura 2. Registro de pensamientos interferentes/no interferentes}

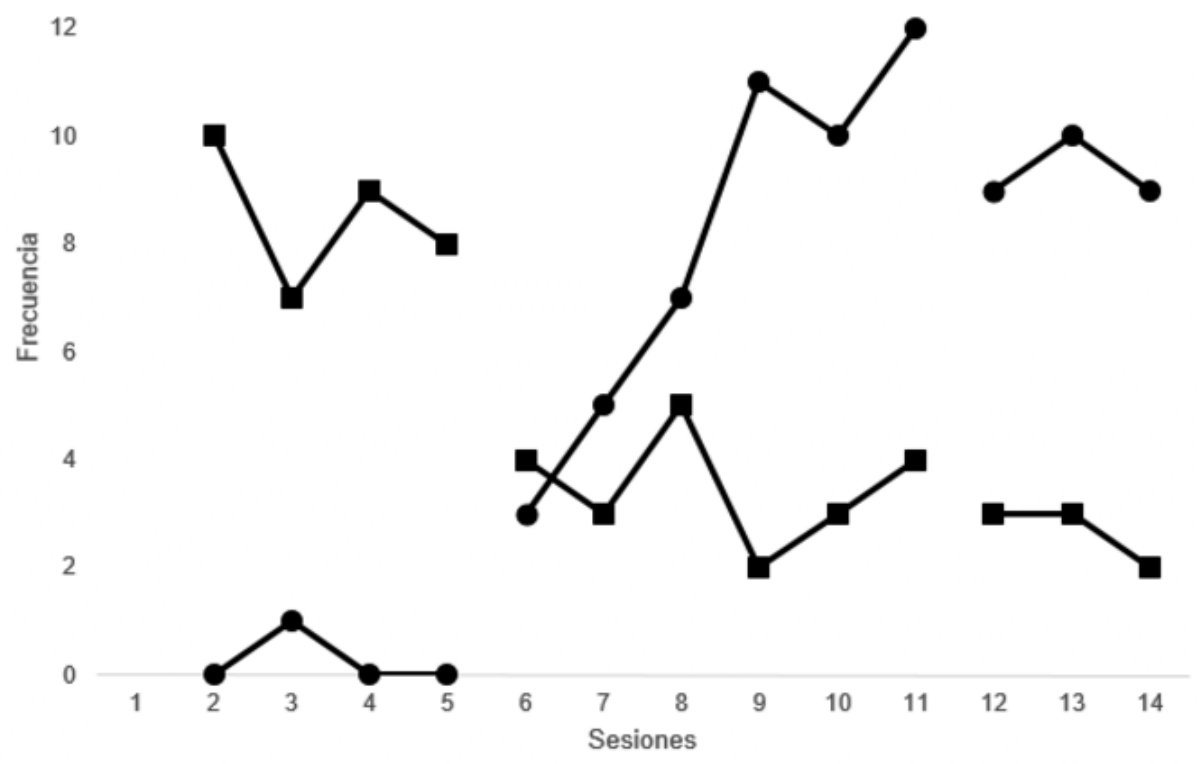


Figura 3. Registro de tareas en las esferas vitales

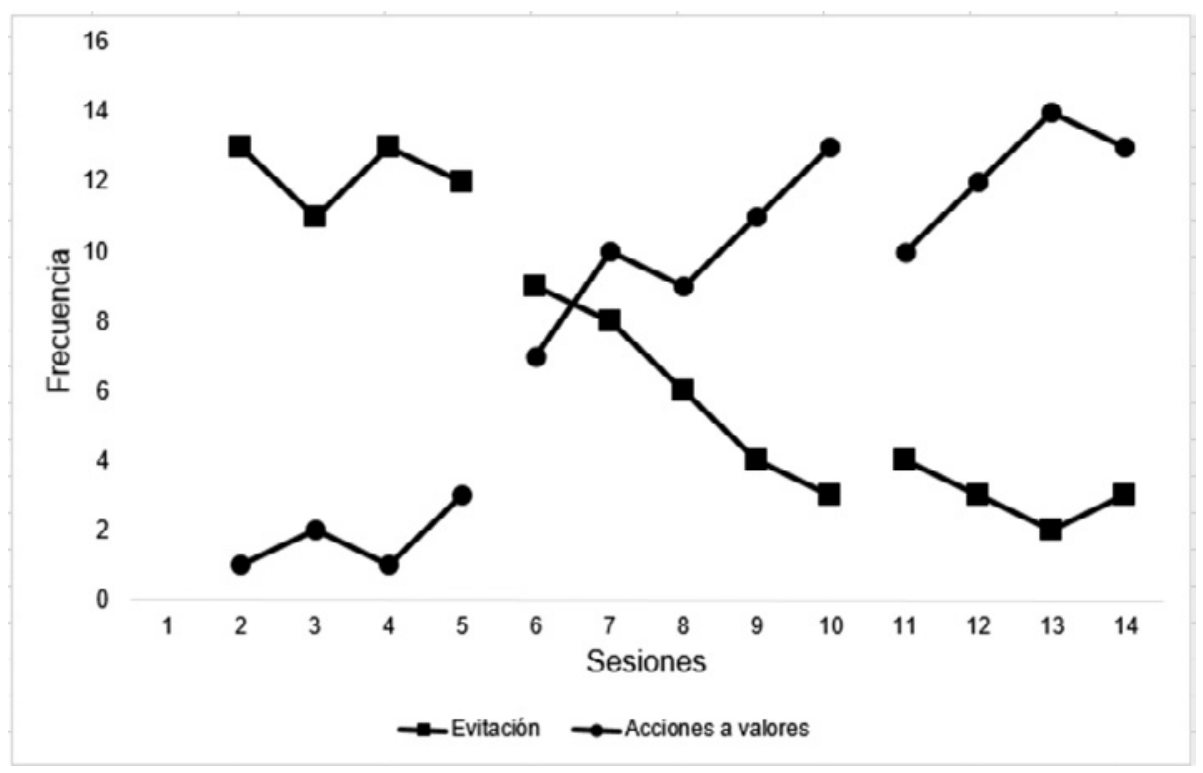

conductual y realizando con mayor constancia las actividades planteadas para las esferas vitales mientras que las conductas de evitación fueron disminuyendo.

\section{DISCUSIÓN}

El abordaje psicológico de pacientes con cáncer se ha venido realizando desde los diferentes enfoques y con una amplia diversidad de estrategias terapéuticas ${ }^{(23)}$. El modelo seleccionado para la intervención del caso aquí presentado es el de la Terapia de Aceptación y Compromiso que fue seleccionado considerando que las conductas problema de la paciente (depresión/ansiedad) se habían cronificado desde la adolescencia hasta la edad actual; la paciente presentaba conductas correspondientes con la "evitación experiencial" y con el control inflexible de sus eventos privados, así mismo, se observaban numerosos mandos verbales que no le resultan adaptativos y le impedían lograr sus objetivos vitales ${ }^{(22)}$.
Según esta propuesta, las conductas desadaptativas en los pacientes con cáncer tienen como propósito la eliminación o reducción parcial de las propias respuestas privadas relacionadas con la enfermedad, a pesar del costo que ello supone en términos de inutilidad y alteración de la vida, porque aunque a corto plazo, den lugar a una reducción parcial de las funciones aversivas, a la larga, terminan provocando la exacerbación y extensión de tales miedos ante numerosas situaciones, viéndose la persona obligada a actuar para eliminarlos, aunque ello suponga una limitación cada vez más pronunciada en la vida en términos de un estancamiento en las facetas personalmente valiosas $^{(24)}$.

Ante esta situación, la pregunta que se plantea es si los eventos privados son intrínsecamente inhabilitantes, es decir, si causan necesariamente comportamientos desajustados y son realmente barreras que les impiden a los pacientes llevar a cabo la vida que desean. Desde el enfo- 
que de ACT, se señala el papel que juega el contexto socio-verbal en la función que llegan a tener los eventos privados y que llegan a enredar a la persona en respuestas de evitación destructivas ${ }^{(25)}$ como se observa en el caso de LP. De acuerdo con Quiñonez y Sandoval ${ }^{(26)}$ los padres depresivos pueden instruccionar "depresivamente" a través de reglas verbales que el niño va haciendo parte de su conjunto de instrucciones, las cuales posteriormente regulan su conducta. En el caso de LP se logró establecer algunos tactos como los siguientes: "no se esfuerce, no hay posibilidades para usted" "es muy difícil ser feliz en esta vida" "nosotros nacimos fue para sufrir ¿no se da cuenta?" "nunca vamos a salir de aquí" "de la gente como nosotros ni dios se acuerda".

Teniendo como base lo anterior, podría afirmarse que cada uno de los objetivos propuestos para el plan de intervención fue cumplido con éxito. Las tareas terapéuticas funcionaron como elementos de exposición que le permitieron a LP experimentar los eventos privados sin dejar de realizar las actividades requeridas y deseadas en sus esferas vitales, esto significa además que la función verbal también se modificó de modo que las "quejas" ya no actuaban como mandos. Otros resultados adicionales que se obtuvieron de la terapia fueron la disminución de los síntomas depresivos y de ansiedad que eran la queja principal de la paciente. Para el caso de la ansiedad podría afirmarse que el cambio de foco atencional, facilitado por las tareas asignadas fue una herramienta fundamental que propició la disminución de las crisis, del mismo modo, las tareas dirigidas a la reducción del control de las conductas verbales y de los eventos privados sobre las crisis de ansiedad, particularmente los pensamientos de catástrofe tuvieron un impacto positivo en el manejo de los síntomas. Este resultado ha sido reportado en investigaciones de caso para trastornos de ansiedad, indicando que la des-literalización y desactivación de funciones verbales y cognitivas es una técnica que reduce la frecuencia de las crisis $^{(27)}$.

Para el caso de los síntomas depresivos, el componente de activación conductual facilitado por la asignación de acciones orientadas a valores, le permitió a la paciente acceder a contextos reforzantes y por lo tanto reducir la frecuencia e intensidad de la sintomatología depresiva, este resultado ha sido observado en otros estudios de caso $^{(28)}$. Así mismo, la desactivación de las funciones de los eventos privados y de la conducta verbal sobre el patrón depresivo, los pensamientos de indefensión y minusvalía, tuvo un efecto positivo en la reducción de dichos síntomas, debido a que LP ya no los utilizaba como justificación para mantener la conducta depresiva, ni como causas de sus estados de ánimo, este resultado también ha sido reportado por otras investigaciones, indicando el rol de la fusión cognitiva en el origen y mantenimiento de la depresión ${ }^{(29)}$.

Finalmente, podría afirmarse que ACT para el caso de LP facilitó un cambio en la forma de reaccionar ante las emociones y pensamientos que le resultaban aversivos posibilitando la aceptación y la búsqueda de nuevos objetivos que le permitieran retomar el curso de su vida. En este sentido, logró dejar atrás la regla culturalmente extendida de intentar eliminar dichos eventos privados. Teniendo en cuenta lo anterior podría afirmarse que ACT es un modelo terapéutico que reporta eficacia terapéutica para pacientes oncológicos que presentan un patrón de evitación experiencial y un patrón conductual inflexible gobernado principalmente por reglas (conducta verbal). Sin embargo, es necesario aclarar que en este contexto de atención clínica es pertinente considerar otras variables como 
los efectos propios de la enfermedad y el tratamiento, por lo que es imprescindible implementar diferentes recursos terapéuticos que se ajusten a cada caso.

\section{REFERENCIAS BIBLIOGRÁFICAS}

1. Mustaffa S, Mohd A, Abu S, Modh R. Effects of cognitive behavior approaches in anxiety for cancer patient undergone chemotherapy. IJFPSS 2012;2:70-3.

2. Schuyler D. Cognitive therapy for adjustment disorder in cancer patients. Psychiatry 2004;1:20-3. Doi: 10.1186/17450179-5-15.

3. Massie M. Prevalence of depression in patients with cancer. J Nal Cancer Inst Mon 2004; 32. Doi: 10.1093/jncimonographs/ Igh014

4. Stark D, House A. Anxiety in cancer patients. Br J Cancer 2000; 83:1261-7. Doi: 10.1054/bjoc.2000.1405.

5. Breibart W, Alici Y. (2012). Evidence-based treatment of delirium in patients with cancer. J Clin Oncol 2012; 30:1206-14. Doi: 10.1200/JCO.2011.39.8784

5. Paiva C. Discussion about the prevalence of mental disorders depending on the different phases of cancer care within the cancer continuum. J Clin Oncol 2015; 33:1516. Doi: 10.1200/JCO.2014.60.0023 .

6. Arias $S$, Murillo R, Piñeros $M$, Bravo $M$, Hernández G, Pardo C, et al. Prioridades de investigación para el control del cáncer en Colombia. Rev Colombiana Cancerol 2007;11:152-64.

7. Baider L, Peretz T, Hadani P, Koch U. Psychological intervention in cancer patients: A randomized study. Gen Hos Psychiatry 2001;23:272-7. Doi:10.1016/S01638343(01)00158-X

8. Campbell C, Campbell L. A systematic review of cognitive behavioral interventions in advanced cancer. Patient Ed Counsel 2012; 89: 15-24.

9. Páez M, Luciano C, Gutiérrez O. La aplicación de la terapia de aceptación y com- promiso (ACT) en el tratamiento de problemas psicológicos asociados al cáncer. Psicooncología 2005;2:49-70.

10. Luciano C, Hayes S. Trastorno de evitación experiencial. Rev Internal Psicol Clíni Salud 2001;1:109-57.

11. Luciano M, Valdivia M, Gutiérrez $O$, Páez M. Avances desde la Terapia de Aceptación y Compromiso (ACT). EduPsykhé 2006;5: 173-201.

12. Páez M, Luciano C, Gutiérrez O. Tratamiento psicológico para el afrontamiento de cáncer de mama. Estudio comparativo entre estrategias de afrontamiento y de control cognitivo. Psicooncología 2007;4:75-95.

13. Twohig M, Crosby J, Enno A.Treatment of three anxiety disorder cases with Acceptance and Commitment Therapy in a private practice. J Cog Psychother: Internal Quart 2011;25:203-17.

14. Montesinos F, Hernández B, Luciano M. Aplicación de la terapia de aceptación y compromiso en pacientes enfermos de cáncer. Análisis y Modificación de Conducta $2001 ; 27: 503-23$.

15. Sturmey P. Behavioral case formulation and intervention. London: Wiley Blackwell, 2008.

16. Smith J. Single-case experimental designs: A systematic review of published research and current standards. Psychol Methods 2012; 17:510-50. Doi: 10.1037/ a0029312.

17. Beck, A. T., Steer, R. A., y Brown, G. K. (2011a). Manual. BDI-II. Inventario de Depresión de Beck-II (Adaptación española: Sanz, J., y Vázquez, C.). Madrid: Pearson

18. Rico J, Restrepo M, Molina M. Adaptación y validación de la escala hospitalaria de ansiedad depresion (HAD) en una muestra de pacientes con cáncer del Instituto Nacional de Cancerología de Colombia. Avances en Medición 2005;3:73-86.

19. Spielberger CD, Gorsuch RL, Lushene RE. Inventario de Ansiedad Estado/Rasgo (STAI). Madrid: TEA Ediciones, 1997. 
20. Barraca J. Spanish adaptation of the acceptance and action questionnaire. Internal J Psychology Psychol Ther 2004;4: 505-15.

21. Wilson K, Luciano M. Terapia de aceptación y compromiso (ACT). Un tratamiento conductual orientado a los valores. Madrid: Ediciones Pirámide, 2011.

22. Almanza-Muñoz, J, Holland J. Psicooncología: Estado actual y perspectivas futuras. Revista del Instituto Nacional de Cancerología, 2000;46:196-206.

23. Feros DL, Lane L, Ciarrochi, J, Blackledge JT. Acceptance and commitment therapy (ACT) for improving the lives of cancer patients: A preliminary study. Psychooncology 2013;22: 459-64. Doi: 10.1002/ pon.2083

24. Hayes S, Hayes L. Some clinical implications of contextualistic behaviorism: The example of cognition. Behav Ther 1991;23: 225-49. Doi: 10.1016/S00057894(05)80383-1
25. Quiñonez R, Sandoval M. Procesos verbales y desórdenes depresivos: Marcos relacionales y seguimiento de instrucciones. Suma Psicológica 1996;3:147-72.

26. Eifert G, Forsyth J, Arch J, Espejo E, Keller M, Lange D. Acceptance and Commitment Therapy for anxiety disorders: Three case studies exemplifying a unified treatment protocol. Cog Behav Pract 2009;16:368-85. Doi:10.1016/j. cbpra.2009.06.001

27. Kanter J, Baruch D, Gaynor S. Acceptance and Commitment Therapy and Behavioral Activation for the treatment of depression: Description and comparison. Behav Anal 2006;29:161-85.

28. Forman EM, Herbert JD, Moitra E, Yeomans PD, Geller PA. A randomized controlled effectiveness trial of acceptance and commitment therapy and cognitive therapy for anxiety and depression. Behav Modif 2007;31:772-99. Doi: 10.1177/0145445507302202 
